\title{
Lidar Based Off-road Negative Obstacle Detection and Analysis
}

\author{
Jacoby Larson and Mohan Trivedi \\ Computer Vision and Robotics Research Laboratory \\ University of California, San Diego \\ La Jolla, California 92093-0434 \\ jacoby.larson@navy.mil, mtrivedi@ucsd.edu
}

\begin{abstract}
In order for an autonomous unmanned ground vehicle (UGV) to drive in off-road terrain at high speeds, it must analyze and understand its surrounding terrain in realtime: it must know where it intends to go, where are the hazards, and many details of the topography of the terrain. Much research has been done in the way of obstacle avoidance, terrain classification, and path planning, but still so few UGV systems can accurately traverse off-road environments at high speeds autonomously. One of the most dangerous hazards found offroad are negative obstacles, mainly because they are so difficult to detect. We present algorithms that analyze the terrain using a point cloud produced by a 3D laser range finder, then attempt to classify the negative obstacles using both a geometry-based method we call the Negative Obstacle DetectoR (NODR) as well as a support vector machine (SVM) algorithm. The terrain is analyzed with respect to a large UGV with the sensor mounted up high as well as a small UGV with the sensor mounted low to the ground.
\end{abstract}

\section{INTRODUCTION}

Unmanned vehicle navigation and obstacle avoidance has had major breakthroughs in the last few years, showing that a vehicle can drive without human intervention in highly controlled desert and urban environments such as was proved in the Defense Advanced Research Projects Agency (DARPA) Grand Challenge and DARPA Urban Challenge. In addition, the Jet Propulsion Laboratory (JPL) has shown that unmanned vehicles "Spirit" and "Opportunity" can navigate through the harshest of off-road environments, Mars, albeit at a very slow pace. Yet UGV autonomy has been difficult to incorporate into a rugged off-road real-time scenario. This technology lag is in large part due to lack of real-time autonomous off-road traversability analysis for unmanned ground vehicles (UGV), including negative obstacle detection at great distances. Military applications for UGVs such as resupply, casualty evacuation, surveillance, and reconnaissance must accommodate off-road terrain based on the warfighting areas in which the US military is currently involved. Accurately representing off-road terrain and analyzing it in real-time is a challenge for most UGV robotic systems and the majority of UGVs operate at slow speeds over relatively flat terrain. A recent report by SSC Pacific [1] concerning the mobility of UGVs for dismounted marines provides a survey and analysis of current robotic technologies and concludes that there are significant weaknesses in each system, especially in the area of mobility in the face of hazardous terrain. This report also identifies that NATO's vital gaps are "moving in all terrain with tactical behavior in nearly all weather conditions" .
There are significant improvements that need to be made in autonomous obstacle detection and avoidance before those higher mission-oriented tasks can be accomplished in the areas of the world the US military is currently fighting, and detecting negative obstacles is an important aspect of the problems that need to be addressed.

\section{RELATED RESEARCH}

Negative obstacles are difficult to detect, especially at long ranges, but methods used have included searching for negative slopes that are too steep or gaps in data that exceed a distance threshold followed by a drop in elevation or a steep uphill slope [2], [3], [4]. JPL uses both a column detector for gaps that exceed a width and height threshold with a region size filter to eliminate negative obstacles that are too short as well as a unidirectional elevation difference detector. In [5], [6], ray tracing is perfomed from the current position of the laser and context-based labeling from occlusions from the ground or positive obstacles are considered while detecting negative obstacles. JPL also has presented a novel method for detecting negative obstacles using thermal signature for night-time detection [7].

Other methods include using aerial image and lidar data, which has been demonstrated [8] to do negative obstacle detection as well, which can detect the bottom of the negative obstacle, which is not always the case from the perspective of a ground robot.

This work can be beneficial to the general intelligent vehicle public, and can be combined with such computer vision safety systems as have been described in [9], [10].

\section{APPROACH}

\section{A. Sensor and Platform Selection}

The negative obstacle detection methods and software that have been developed for this research are designed for an unmanned ground vehicle using a 3D lidar. This research was conducted to fit both a large and a small unmanned ground vehicle platform, using a large and a small 3D lidar. The intended large platform is a Max ATV (Figure 2). It is a six-wheel skid-steer all terrain vehicle with dimensions of length $2.6 \mathrm{~m}$, width $1.5 \mathrm{~m}$, and height, including a roll bar, of $1.7 \mathrm{~m}$. The 3D lidar used on the large UGV platform is a Velodyne HDL-64E. This lidar system provides readings of range and intensity out to a distance of 120 meters with $80 \%$ reflectivity, providing 100,000 data points with 360 degrees 


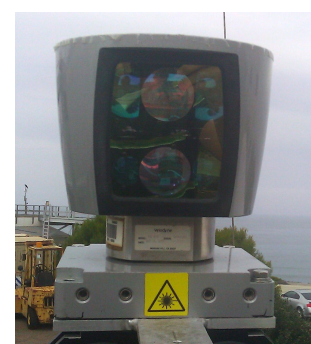

Fig. 1: Velodyne HDL-64E lidar sensor

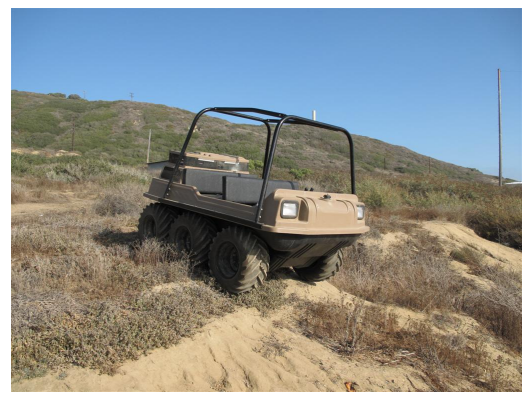

Fig. 2: Max ATV UGV test-bed platform

horizontal and 26.8 degrees vertical field of view at a rate of $10 \mathrm{~Hz}$. SSC Pacific has demonstrated the accuracy of this sensor on the water surface by detecting a lobster trap out to $40 \mathrm{~m}$ and a partially submerged black rock out $65 \mathrm{~m}$ [11]. The smaller ground vehicle platform is an iRobot Packbot (Figure 4) with length $89 \mathrm{~cm}$, width $52 \mathrm{~cm}$, and height $18 \mathrm{~cm}$, mounted with a Hokuyo UTM-30LX lidar sensor, set in a mechanism that tilts it vertically up and down for a full 3D scan, which we refer to as the Nodding Hokuyo (Figure 3). The Nodding Hokuyo scans 270 degrees horizontally and can rotate slow enough to have a 2.5 degree vertical angular resolution, with a pitch from -90 degrees to +90 degrees.

\section{B. Negative Obstacles}

Negative obstacles are ditches or terrain with a steep negative slope that if traversed would be a hazard to the vehicle. Negative obstacles can be just as hazardous to unmanned vehicles as obstacles above ground because they could cause roll-over, tip-over, or high-centering. Usually

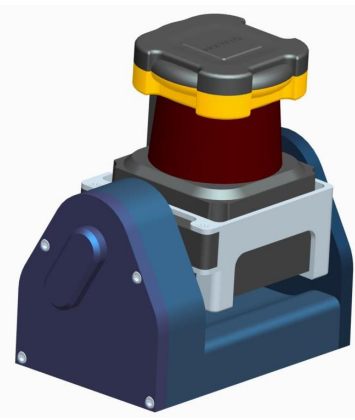

Fig. 3: Computer generated model of Hokuyo UTM-30LX lidar sensor in vertically rotating mechanism, built at SSC Pacific (known as the Nodding Hokuyo)

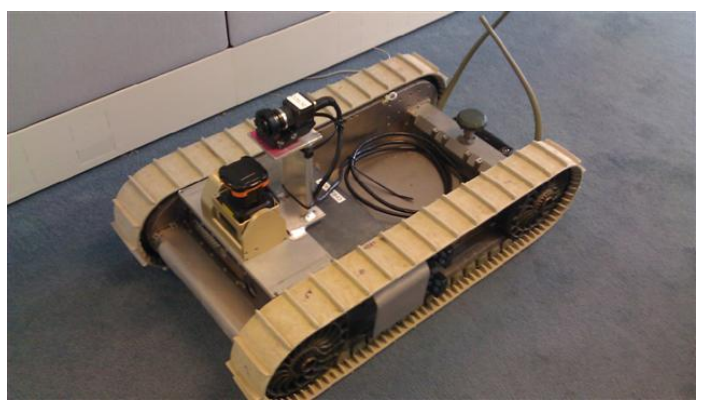

Fig. 4: iRobot Packbot UGV test-bed platform with Nodding Hokuyo sensor

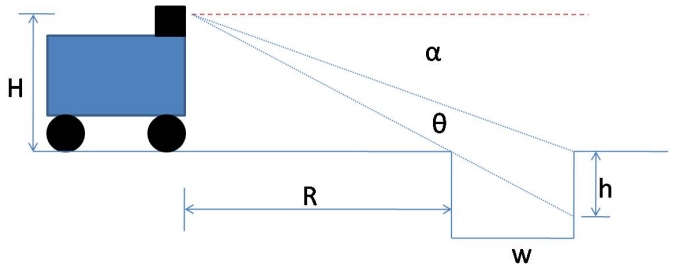

Fig. 5: Geometry of negative obstacle (ditch) detection

ditches that are larger than the width of the diameter of the wheel are enough to cause damage to a vehicle. Obstacles of greater widths may be crossed by vehicles at high enough speeds, but we will not be attempting to provide navigation techniques in this paper, simply methods of detection.

1) Detection Range and Stopping Distance: These hazards are difficult to detect from close up and nearly impossible from far away. Equation 1, based upon the small angle approximation and referenced from [7], illustrates the difficulty of detecting negative obstacles at a range $R$ and is shown in Figure 5. The width of the obstacle is $w, H$ is the height of the sensor from the ground, $h$ is the depth of the obstacle seen by the sensor, and $R$ is the range from the sensor to the obstacle. The equation to solve for $\theta$ is

$$
\theta \approx \frac{H w}{R(R+w)}
$$

The angle $\theta$ decreases significantly as the range increases $\left(\sim \frac{1}{R^{2}}\right)$, which makes negative obstacles so difficult to detect as range increases. Yet detecting negative obstacles at greater distances is essential, especially for fast moving UGVs. Two different methods to detect negative obstacles are used in this paper. The first method uses a support vector machine (SVM) that must be trained with ground-truth data. It has been expected that there would be a limit in the range of correct classification for the SVM because of the parameters passed into it. The second method is called the Negative Obstacle DetectoR (NODR), which uses a number of filters and looks for contextual cues, so it can have expanded range benefits.

It is known from [12] that the stopping distance for a vehicle can be determined using Equation 2.

$$
R=\frac{v^{2}}{2 \mu g}+v T_{r}+B
$$


where $\mu$ is the coefficient of static friction between ground and wheels with a common value of 0.65 for off-road driving, $g$ is gravitational acceleration with a value of $9.8 \mathrm{~m} / \mathrm{s}^{2}, T_{r}$ is the total reaction time with a common value of $0.25 \mathrm{~s}$, and $B$ is a buffer distance used for safety with a value of $2 m$ in our experiments. The velocity value becomes the dominant term at $v>3.2 \mathrm{~m} / \mathrm{s}$ : for a velocity of $24 \mathrm{kph}$, the distance needed to stop is $7.2 \mathrm{~m}$; for a velocity of $48 \mathrm{kph}$, the distance needed to stop is $19.4 \mathrm{~m}$.

2) NODR Classification Approach: Because of the difficulty in detecting negative obstacles, this classification method errs on the side of generously detecting negative obstacles and then labeling them as only potential negative obstacles. Only when the potential obstacle comes within close range to the vehicle can enough data be gathered to truly classify it as a real negative obstacle. This geometry-based method for detecting negative obstacles is called Negative Obstacle DetectoR (NODR) and is shown in Figure 8.

The NODR classifies potential negative obstacles by detecting gaps, an absence of data, where there could exist a ditch, cliff, or negative slope. The detection starts by tracing a ray of $3 \mathrm{D}$ points outward from the sensor, following the returns from the vertical alignment of lasers starting with the lowest vertical angle towards the highest angle. This algorithm is based on laser sensors that produce structured results, such that can be vertically aligned. The Hokuyo UTM-30LX returns a maximum of 1080 lidar beams per horizontal scan and the vertically rotating mechanism allows the Hokuyo to produce another horizontal scan above or below the previous scan. This aligns well for following a vertical ray of lidar beams without any modification. However,the output from the Velodyne HDL-64E needs to be modified slightly. This sensor has 64 vertically aligned lasers but reports 3 laser pulses in the top 32 lasers for every 1 pulse in the bottom 32 lasers. To handle the ray tracing in this case, we have combined the 3 laser returns from the top 32 lasers into one return by taking the average $\mathrm{x}, \mathrm{y}$, and $\mathrm{z}$ values of the top 32 lasers and using the default values of the bottom 32 lasers, providing one complete ray of 64 lasers.

The first step used in the NODR method is to search for a step, a drop in elevation beyond a step threshold, that extends beyod the gap distance. The gap threshold of our two UGVs are determined by the size of a hole that would cause damage or stop the platform. For those vehicles with wheels, it is usually the size of the diameter of a wheel. For the Max ATV with 3 wheels on each side, and the tracked vehicles like the iRobot Packbot, it is the distance from the front wheel or tread to the center of gravity. Note: no center of gravity measurments were taken for either of these two ground vehicles and the gap threshold values used were only estimates for the simulated environment.

If the step search does not return a negative obstacle, then the NODR looks for gaps in data between two points. While tracing these radial rays, if a gap is found with a distance greater than a gap threshold then it looks at the gap angle. The gap angle filter uses the increase in vertical angular resolution between the two scans. The threshold here is the distance that would be expected if the next point had the same elevation as the previous point, and the vertical angle had increased by $\gamma * \Delta$ where $\Delta$ is the vertical angular resolution between horizontal scans (Figure 6). Tests provided the best results when $\gamma$ had a value of 1.5. It is noted that this might not be the most accurate method to calculate true negative obstacles, but our algorithm is attempting to be conservative to avoid obstacles, and as was emphasized from Equation 1, negative obstacles are extremely difficult to detect at long ranges. Furthermore, if a gap is sufficiently wide to pass these distance filters, the algorithm looks for contextual cues.

The cues to look for are either a significant drop in elevation, such as one that would appear if the slope was steeper than our maximum declining slope threshold (Figures $7 \mathrm{a}$ and $7 \mathrm{~b}$ ), or the data after the gap has a significant positive slope (Figure 7c), as in the sloping up-side of a ditch. The slope of the up-side (the back) of a ditch can be determined by first calculating the number of following points should be part of the back slope, determining their slope, and threshold that value. The number of points is calculated by finding the viewing angle of the negative obstacle. In Figure 5, this is $\theta$. For ranges $\mathrm{R}$ that are not quite so distant, where the small angle approximation doesn't work and Equation 1 doesn't provide an accurate measurement of $\theta$, this equation is

$$
\theta=\tan ^{-1}\left(\frac{H}{R}\right)-\tan ^{-1}\left(\frac{H}{R+w}\right)
$$

The number of slope points to be used is num_points $=\frac{\theta}{\Delta}$, where $\Delta$ is the vertical angular resolution of the sensor. As long as num_points is greater than 1, we can determine the back slope. When the gap is detected at ranges where a back slope is impossible to calculate because the vertical angle resolution is less than $\theta$, it is simply classified as a potential negative obstacle.

Our research concluded that it does not add value to differentiate between the gaps caused by a true negative obstacle or just occlusion from a positive obstacle. A vehicle should not navigate through a positive obstacle anyway, and processing the potential negative obstacles is trivial, whereas detecting positive obstacles and removing potential negative obstacles found afterwards can take away many clock cycles of precious processing time.

The result from the NODR is a vector ray between two laser points that can be used to populate a grid map of obstacles and terrain features. The approach we have taken in our research is not to completely avoid these detected obstacles, because at this point they are only potential negative obstacles. They could still be steep slopes that are traversable but our sensors do not have complete information because of the proximity to the vehicle and limitations of our geometry-based methods. In most cases the vehicle must be much closer to actually classify these as true negative obstacles. Our proposed method is to slow down when these potential negative obstacles are in the immediate navigable path, and avoid them once they have been truly classified. 


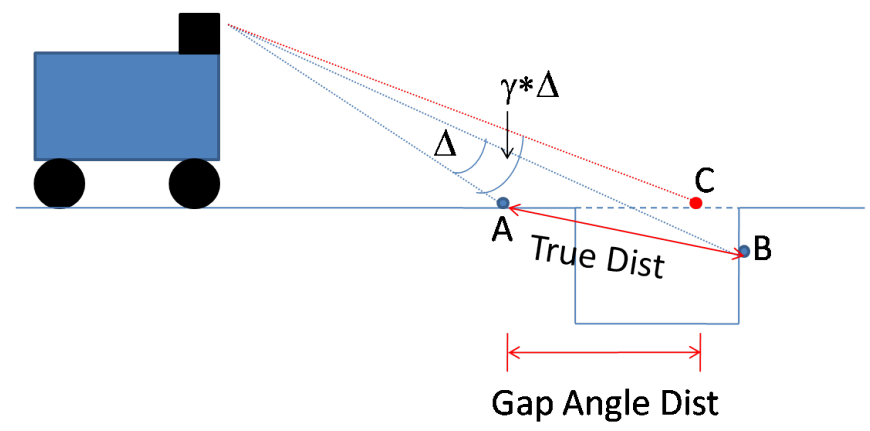

Fig. 6: A potential negative obstacle will have a gap with a distance greater than if the next laser point was found on a flat surface with an increase in angle of $\gamma$ times the angle difference. Points A and B are true returns from a lidar traced along a ray. The expected increase in vertical angle is $\Delta$. If the surface was flat and the vertical angle was $\gamma * \Delta$, the next laser return would have been $\mathrm{C}$. If the distance between $\mathrm{A}$ and $\mathrm{B}$ is greater than $\mathrm{A}$ and $\mathrm{C}$, this could be a negative obstacle.

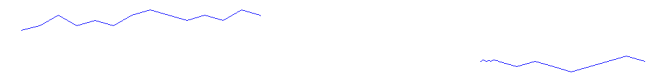

(a) Gap followed by a large drop in elevation: potential negative obstacle
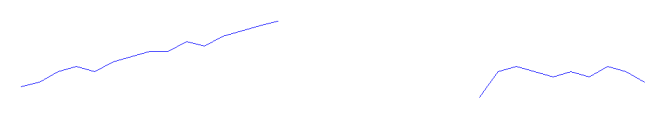

(b) Slight uphill slope followed by a gap and drop in elevation: potential negative obstacle

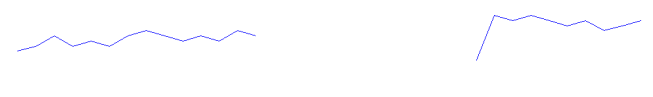

(c) Gap followed by a steep uphill slope: potential negative obstacle

Fig. 7: Ray tracing examples with results determined during the potential negative obstacle detection step

\section{SVM Classification Approach}

As with the NODR classification approach, the support vector machine returns the vector rays in between laser points that are classified as potential negative obstacles. Parameters passed into the SVM include the range to the first point (of a pair of two vertically aligned points) from the sensor, the distance between the two points, the change in vertical angle of the two points (as if the second point had the same elevation value as the first point) in reference to the vertical angular resolution, and the elevation difference of the two points. The SVM is trained by many samples of ground truth vector rays of negative obstacles as well as non-negative obstacles, then takes in each pair of vertically aligned points from the test cases to determine its classification.

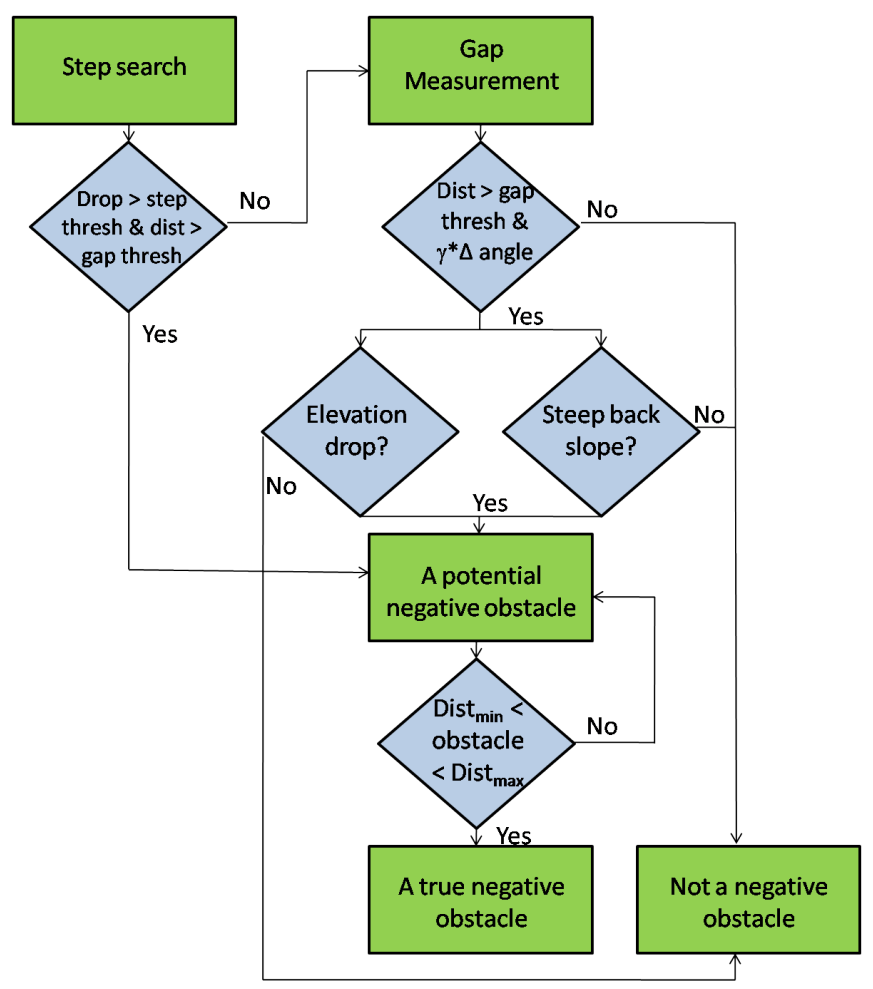

Fig. 8: Negative Obstacle DetectoR (NODR) flow chart

\section{Real Negative Obstacle Classification}

Those potential negative obstacles that are within a narrow range of values are elevated to a true negative obstacle hazard condition. This range is calculated based on the maximum negative slope the vehicle can traverse and the vertical angle measurements from the sensor, found in Equation 4 and in Figure 9. For instance, the maximum negative slope we are willing to let both of our vehicles traverse, $\phi$, is a 20 degree decline. Based on the maximum negative vertical angle, $\theta_{\max }$, of the sensor and the angle between each increasing horizontal scan, $\Delta$, we would only be able to detect a steeper decline between Dist $t_{\text {min }}$, and Dist $t_{\max }$. For the Velodyne on the Max ATV, this range is between 4.76 meters and 5.91 meters. After Dist $t_{\max }$, the angle of the lidar scan with the horizontal is less than $\phi$ and would not actually detect the slope. Therefore, those potential negative obstacle rays that begin between Dist $_{\min }$ and Dist max $_{\text {are considered real }}$ negative obstacles. This is a very short distance to react to a real negative obstacle, but the navigation module should have already slowed down for the potential negative obstacles as it was approaching them (the speed should be slow enough to allow stopping distance before the obstacle, using Equation 2).

$$
\begin{aligned}
\text { Dist }_{\min } & =\frac{H}{\tan \left(\theta_{\max }\right)} \\
\text { Dist }_{\max } & =\frac{H}{\tan \left(\theta_{\min }\right)} \\
\theta_{\max }, \theta_{\min } & >\phi
\end{aligned}
$$




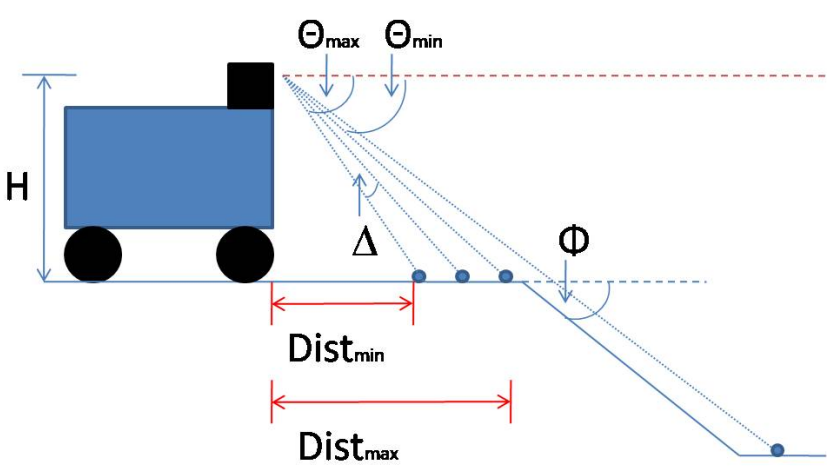

Fig. 9: Sensor angles used to find the min and max distance a steep negative decline can be discovered.

\section{EXPERIMENTAL EVALUATION}

The detection methods follow a vertical alignment of ladar beams and report the vector ray between two beams where it has detected a negative obstacle. For each negative obstacle in a simulated terrain map there will be multiple negative obstacle rays. Because each of the terrains were created from a simulation, the exact location and vector ray is known and is labeled as ground truth. If the detection methods finds a ray that overlaps one of the ground truth rays, it is considered a positive ray detection. Those detected rays that do not match up with a ground truth ray are considered a false ray detection. If there exists even one positive ray detection for a negative obstacle, this is counted as a positive obstacle detection. The results of negative obstacle ray detections as well as negative obstacle detections are reported in this paper.

\section{A. Setup of Simulated Environment}

To better analyze negative obstacles and their various representations, multiple simulated scenes were created, filled with negative obstacles that would cause damage or halt the robotic vehicle. These scenes were created by first building a height-map of the terrain with any number of negative and positive obstacles with specified sizes, as well any value of roughness for the rest of the terrain. The roughness was set by randomly selecting height values from a Gaussian distribution. To simulate the lidar returns from the terrain, a vector ray was created from the virtual location of the sensor on the height-map (depending on the size of the simulated platform), and a height-map intersection algorithm was used to simulate a lidar pulse from the sensor. This was performed by determining when the $\mathrm{z}$ value of the initial location of the lidar ray switches between "above" to "below" the terrain, or vice versa; comparing $\mathrm{z}$ values of the ray vector with the $\mathrm{z}$ values of the terrain heightmap at the same $\mathrm{x}, \mathrm{y}$ location. The $\mathrm{x}, \mathrm{y}$, and $\mathrm{z}$ location where the lidar ray crosses over becomes the $3 \mathrm{D}$ point to be added to the point cloud list. The simulated 3D point cloud of the terrain was obtained by following the horizontal and vertical angular resolution pattern of both the small and large 3D lidar sensors that were used in this research (see Figure 10).

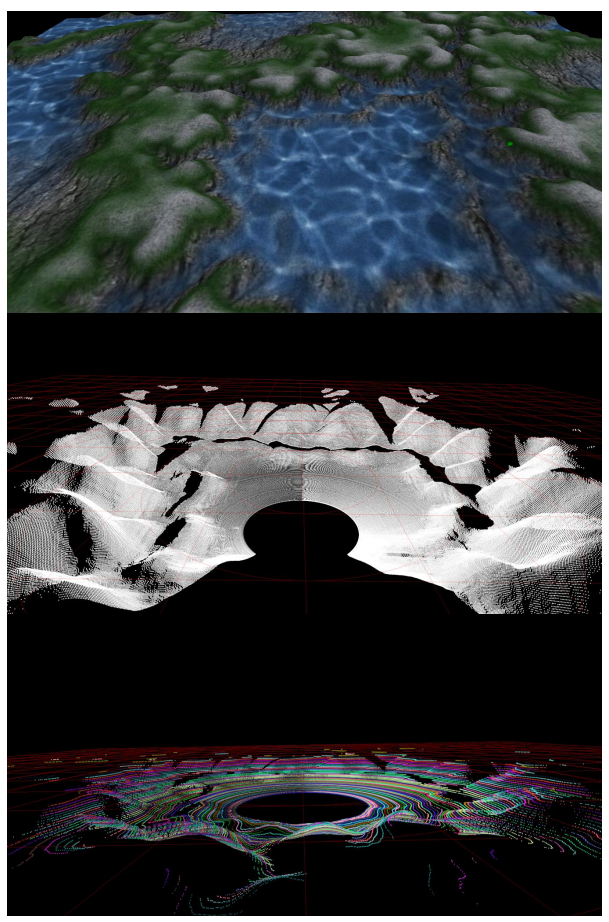

Fig. 10: Simulated sample terrain (top) with lidar scans from both the small (middle) and large (bottom) 3D lidars

\section{B. Selecting of Test Cases}

For this experiment, the simulations set the height of the lidar appropriate for their vehicle platforms

Four experiments were conducted, two simulating the small lidar and two for the large lidar in a combination of smooth and rough terrain, separating results for the NODR and SVM methods. All of the simulated terrains had negative obstacles and some had randomly placed positive obstacles as well.

The SVM was trained with multiple sample height maps of equal amounts ground truth negative obstacle rays and non-negative obstacle rays, then tested with new simulated obstacle maps.

\section{Results of Experiments}

Table I displays the results of the overall negative obstacle rays and negative obstacles detected. Negative obstacles are detected when one negative obstacle ray is detected inside a negative obstacle. The results from each method are the combination of of results from both simulated rough and smooth terrains. The range of the experiment describes the range at which obstacles were placed for that experiement. The SVM experiments were extremely accurate within a short range, which was even shorter for rougher terrain. A point cloud representation from the small lidar with negative obstacle detection images of the rough test case is depicted in Figure 11. Beyond the short range, the SVM method innacurately identified every single ray beyond as negative obstacles, which can be seen in Figure 12. Our hypothosis is that the negative obstacles begin to be smaller than the length between vertical lidar pulses and the SVM cannot gather enough information to make a proper decision. 
TABLE I: Lidar Experiment Results

\begin{tabular}{lllll} 
Lidar & Method & Range & $\begin{array}{l}\text { Neg Obs Rays } \\
\text { Detected }(\%)\end{array}$ & $\begin{array}{l}\text { Neg Obs De- } \\
\text { tected }(\%)\end{array}$ \\
\hline Small & NODR & $30 \mathrm{~m}$ & 52 & 78 \\
Small & SVM & $6-8 \mathrm{~m}$ & 98 & 100 \\
Large & NODR & $50 \mathrm{~m}$ & 27 & 31 \\
Large & SVM & $16-20 \mathrm{~m}$ & 53 & 89 \\
\hline
\end{tabular}

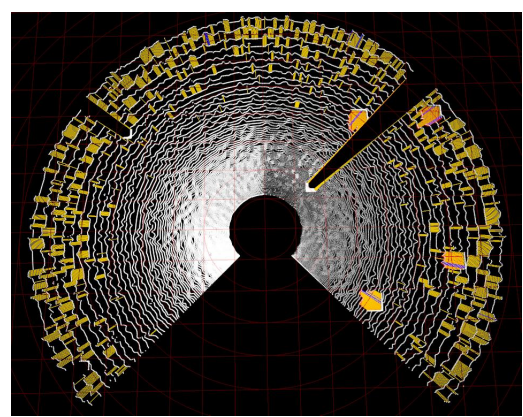

Fig. 11: Detection image for the rough SVM experiment for the small lidar. The gaps in data are the negative obstacles, and most are colored by the detection method (SVM detection is in yellow). There are positive obstacles as well, and all the data behind it is occluded from the lidar. Beyond the short range limit $(6 \mathrm{~m})$ there tends to be much more noise.

\section{CONCLUSION AND Future WORK}

Based on the results from these experiments and according to the stopping distance equations, the small vehicle can travel $2.5 \mathrm{~m} / \mathrm{s}$, which is its maximum speed, process the data in $0.5 \mathrm{~s}$ (our software can process negative obstacles as well as 3D traversability analysis and path planning at an average rate of $2 \mathrm{~Hz}$.), and still stop $2.2 \mathrm{~m}$ away, far away from the SVM rough terrain range limit of $6 \mathrm{~m}$. As well, the large vehicle can travel $37 \mathrm{kph}$, process the data in $0.5 \mathrm{~s}$, and stop $14 \mathrm{~m}$ away, just shy of the svm rough terrain range limit of $16 \mathrm{~m}$. This is a good benchmark to start from for the goal of high-speed off-road autonomous driving.

The best solution for negative obstacle detection is a combination of SVM for the short range and NODR for long range detection. Understanding this exact range limit and how to push it out even further is a task for future work and could provide even better results.

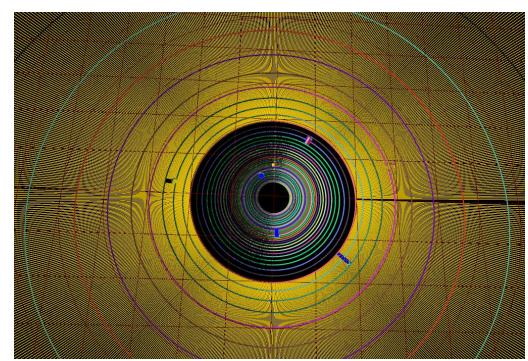

Fig. 12: False positives (yellow) reported for ranges that were beyond the short range limit $(16 \mathrm{~m})$ for the simulated large lidar sensor
It has been demonstrated that it is possible to analyze terrain data, classifying hazards such as positive obstacles, steep slopes, step edges, and even negative obstacles at a distance far enough to travel at relatively high-speeds. This work will be beneficial for autonomous UGV military and commercial applications in off-road terrain.

One of the issues of attempting to detect negative obstacles at long ranges is the vertical angular resolution of the 3D lidar. A possible solution to improving this angular resolution is to retain multiple scans and analyze them as the vehicle is moving, either from the 3D lidar or even a single-scan lidar, angled in such a way that it would find negative obstacles far enough away to react in time. The distance between consecutive scans would be dependent only on the refresh scan rate of the lidar and the speed of the vehicle. For instance, a lidar scanning at $40 \mathrm{~Hz}$ on a vehicle traveling at $32 \mathrm{kph}$ can detect the ground every $0.22 \mathrm{~m}$ (which can be set any distance away from the vehicle). Currently the smaller lidar skips $2 \mathrm{~m}$ between lidar scans at $16 \mathrm{~m}$ away. The large lidar skips $32 \mathrm{~m}$ between lidar scans $75 \mathrm{~m}$ away.

\section{REFERENCES}

[1] S. Holste and D. Ciccimaro. Increasing the mobility of dismounted marines. Technical Report 1988, SSC Pacific, 2009.

[2] H. Seraji. Rule-based traversability indices for multi-scale terrain asessment. In Proceedings of 2003 IEEE International Conference on Control Applications, Istanbul, Turkey, June 2003.

[3] A. Murarka, M. Sridharan, and B. Kuipers. Detecting obstacles and drop-offs using stereo and motion cues for safe local motion. In International Conference on Intelligent Robots and Systems (IROS), 2008.

[4] A. Rankin, A. Huertas, and L. Matthies. Evaluation of stereo vision obstacle detection algorithms for off-road autonomous navigation. In AUVSI Symposium on Unmanned Systems, 2005.

[5] T. Hong, S. Legowik, and M. Nashman. Obstacle detection and mapping system. National Institute of Standards and Technology (NIST) Technical Report NISTIR 6213, pages 1-22, 1998.

[6] N. Heckman, J-F. Lalonde, N. Vandapel, , and M. Hebert. Potential negative obstacle detection by occlusion labeling. In Proceedings of International Conference on Intelligent Robots and Systems (IROS), 2007.

[7] L. Matthies and A. Rankin. Negative obstacle detection by thermal signature. In Proceedings of 2003 IEEE/RSJ International Conference on Intelligent Robots and Systems (IROS), pages 906-913, 2003.

[8] D. Silver, B. Sofman, N. Vandapel, J. Bagnell, and A. Stentz. Experimental analysis of overhead data processing to support long range navitation. In International Conference on Intelligent Robots and Systems, 2006.

[9] M.M Trivedi, T. Gandhi, and J. McCall. Looking-in and looking-out of a vehicle: Computer-vision-based enhanced vehicle safety. In IEEE Transactions on Intelligent Transportation Systems, pages 108-120, March 2007.

[10] T. Gandhi and M.M. Trivedi. Vehicle surround capture: Survey of techniques and a novel omni video based approach for dynamic panoramic surround maps. In IEEE Transactions on Intelligent Transportation Systems, September 2006.

[11] R. Halterman and M.H. Bruch. Velodyne hdl-64e lidar for unmanned surface vehicle obstacle detection. In SPIE Proc. 7692: Unmanned Systems Technology XII, pages 644-651, Orlando, FL, April 2010.

[12] L. Matthies and P. Grandjean. Stochastic performance modeling and evaluation of obstacle detectability with imaging range sensors. IEEE Transactions on Robotics and Automation, 16(12), December 1994. 